\title{
Ethnotaxonomy of birds by the inhabitants of Pedra Branca Village, Santa Teresinha municipality, Bahia state, Brazil
}

\author{
Ana Teresa Galvagne Loss ${ }^{1 *}$, Eraldo Medeiros Costa Neto ${ }^{2}$, Caio Graco Machado ${ }^{2}$ and Fernando Moreira Flores ${ }^{1}$
}

\begin{abstract}
Background: Studies on popular names of birds help to understand the relationship between human beings and birds and it also contributes to the field of ornithology.

Methods: This study aims to register the ethnotaxonomy of birds in the village of Pedra Branca, Santa Teresinha municipality, Bahia State, Brazil, by cataloguing and identifying their popular names, besides understanding the ethnoclassification system of local bird species. The ethno-ornithological data were obtained by means of semi-structured open interviews, and projective tests.

Results: We interviewed 48 residents and, in order to identify species, we chose five informants with a more detailed knowledge on local avifauna. We registered 139 common names, distributed into 108 ethnospecies and 33 synonyms, referring to 117 species. Nomenclatural criteria more frequently used were vocalization and coloring patterns. Following Berlin's principles of ethnobiological classification, three hierarchical levels were registered: life form, generic and specific, with three types of correspondence between Linnaean and folk classification systems. The bird life form ("pássaro" in Portuguese) was associated only to wild species.

Conclusions: The ethno-ornithological research in Pedra Branca Village has contributed with new information on popular nomenclature of birds and their etymology, showing that folk knowledge on birds is conveyed within the community.
\end{abstract}

Keywords: Human beings, Bird, Ethno-ornithology, Local names, Hierarchical levels, Vocalization, Ethnoetymology

\section{Introduction}

Studies on biological ethnotaxonomy aim at investigating how living organisms are perceived, identified, named and classified, seeking to understand how people categorize (ethnosemantic domains) and organize (ethnotaxonomic structures) nature elements [1-4]. Some ethnobiological principles of classification and nomenclature aim to identify similarities between cognitive systems in various societies. So, it is important to find out what are the ethnoclassification criteria (morphological, ecological, ethological, etc.), in order to develop a representative taxonomy of the classification system within a certain community [5,4,6-8]. Ethnobiological classification may

\footnotetext{
* Correspondence: anatebio@yahoo.com.br

${ }^{1}$ Post-Graduation Program in Zoology, Department of Biological Sciences, Feira de Santana State University, Feira de Santana, Bahia 44036-900, Brazil Full list of author information is available at the end of the article
}

be a good indicator of the cognitive and behavioral language process [9].

Considering that human beings, on different parts of the world, use similar cognitive strategies to classify living things and organize biological concepts, studies on ethnotaxonomy show, in fact, that the main problem has always been finding what are the similarities or differences that could be really important for classification purposes [10-13].

Among current animal species, birds draw attention due to their gorgeous coloring and shrill songs [14]. Cooker [15] registered the names attributed by the Chippewa Indians, in northern Mexico, to birds from their region, reporting those they used; this investigation resulted in the first ethno-ornithological study, titled Bird nomenclature of the Chippewa Indians. Regarding the origin of names, the author observed the use of 
morphological criteria and habitats, and there were also names of species with no meaning.

Whereas the scientific nomenclature of birds has been established for over 200years, popular and vernacular designation, as a product of people's imagination, has no systematization. Vernacular names are popular, vulgar, or common names, which are names adopted by people who live in the regions inhabited by birds [14]. Thus, investigating vernacular names of birds from a certain region provides ornithology with the possibility of registering new occurrences, describing unknown behaviors, locating endangered species, and pointing out conservation alternatives, as well as understanding the relationships between human beings and birds, explaining to society the intrinsic value of cultural diversity [16].

In Brazil, investigations and contributions from local knowledge on birds started when settlers first came to Brazil; they registered bird popular names, as well as stories and legends told by native people, which served, since the beginning, as data on the Brazilian avifauna [7]. Later, many studies were conducted registering popular names and the reproductive ecology of several species [17]; descriptions of customs, superstitions, and Brazilian and American legends, addressing the etymology of some names [18]. Sick [14], who is a reference in ornithology, deal with vocalization transcripts, etymology of bird names, and in a very summarized way, legends involving some species.

Guided by a theoretical framework of cognitive anthropology, there are the following works: Jensen [19] examined bird classification systems among four Indian groups having similar environments and lifestyles in the Amazon: Wayampi, Urubu-Ka'apor, Sateré-Mawé, and Apalaí; Giannini [20] investigated, along with Xicrin Indians, the existence of an Indian ethnoclassification of the avifauna from the Rio Cateté region, in Pará, Brazil; and Carrara [21] examined the ethnobiological classification of Xavante Indians in Mato Grosso, Brazil.

The present article registers the ethnotaxonomy of birds known by residents of the village of Pedra Branca, in the municipality of Santa Teresinha, Bahia, Brazil, cataloguing and identifying popular names, with their etymological description, besides analyzing the ethnoclassification system of local bird species. The biological ethnotaxonomic study is of great importance both for understanding and grasping local biodiversity and investigating the universality of human ability to classify the biological world.

\section{Materials and methods}

\section{Study area}

The village of Pedra Branca is located in the municipality of Santa Teresinha (12 $44^{\prime} 30^{\prime \prime} \mathrm{S}$ and $\left.39^{\circ} 34^{\prime} 50^{\prime \prime} \mathrm{W}\right)$, in the central-west area of Bahia State, Brazil, a region with subhumid to dry climate features (Figure 1). This municipality has a population of 9,648 people and it is $192 \mathrm{~km}$ far from the state capital city, Salvador [22,23].

Pedra Branca has 406 residents, with 136 families enrolled in the local health care unit and higher concentrations in the age groups from 20 to 39 years and above 60 years. Local agriculture is based on cassava crop (Manihot esculenta Crantz, Euphorbiaceae) and grape crop for producing red wine; livestock is related to cattle breeding. Men sometimes have jobs in civil construction [24,25,23].

The village lies at the bottom of a mountainous massive known as Serra da Jiboia, which has around 22,500 ha in area and a maximum altitude of $850 \mathrm{~m}$. Serra da Jiboia is located southern Recôncavo region in Bahia and comprehends the territories of five municipalities: Castro Alves, Elísio Medrado, Santa Teresinha, São Miguel das Matas, and Varzedo [26-28].

Serra da Jiboia is located in an ecotone zone, between the ecosystems of Atlantic Forest and Caatinga, which provides it with a great diversity of climates, reliefs, soils, vegetation, and fauna, being one of the most western sites of the Atlantic Forest in Bahia and one of the wettest forests in the most northern hillside of the state. Its climate ranges from humid tropical, at east and southeast, to sub-humid tropical, at west and northwest [28]. Floristic studies in the area report the occurrence of many plant formations with rock fields on the top, Caatinga at the bottom, and hygrophilous forest at the slopes [29-32]. Regarding avifauna, two studies are known for the region, on distinct areas of Serra da Jiboia, where 221 species were registered by Freitas and Moraes [33] and 233 species were registered in surveys conducted by the Feira de Santana State University (UEFS) (PhD Caio Graco Machado, person. commun.). The richest region comprises areas where Pedra Branca inhabitants perform some of their daily activities.

\section{Data collection and analysis}

Field collection was conducted within the period from August 2011 to June 2012 and 48 residents of both sexes were interviewed, 24 men and 24 women, aged from 18 to 87 years. A free and informed consent term (Resolution 196/1996 from the Ministry of Health) has been prepared to explain the objectives of this study; it was distributed to participants, asking if they agreed to provide information, respecting the decision of those who declined to participate in the research. The study was approved by the Research Ethics Committee of UEFS (CAAE 0077.059.000-11).

Initially, data were obtained both through open interviews, exploring and detailing the theme under study, and semi-structured interviews based on a list of preselected topics. As a final phase of data collection, a projective test was performed consisting on the presentation of visual and auditory resources to encourage interviewees 


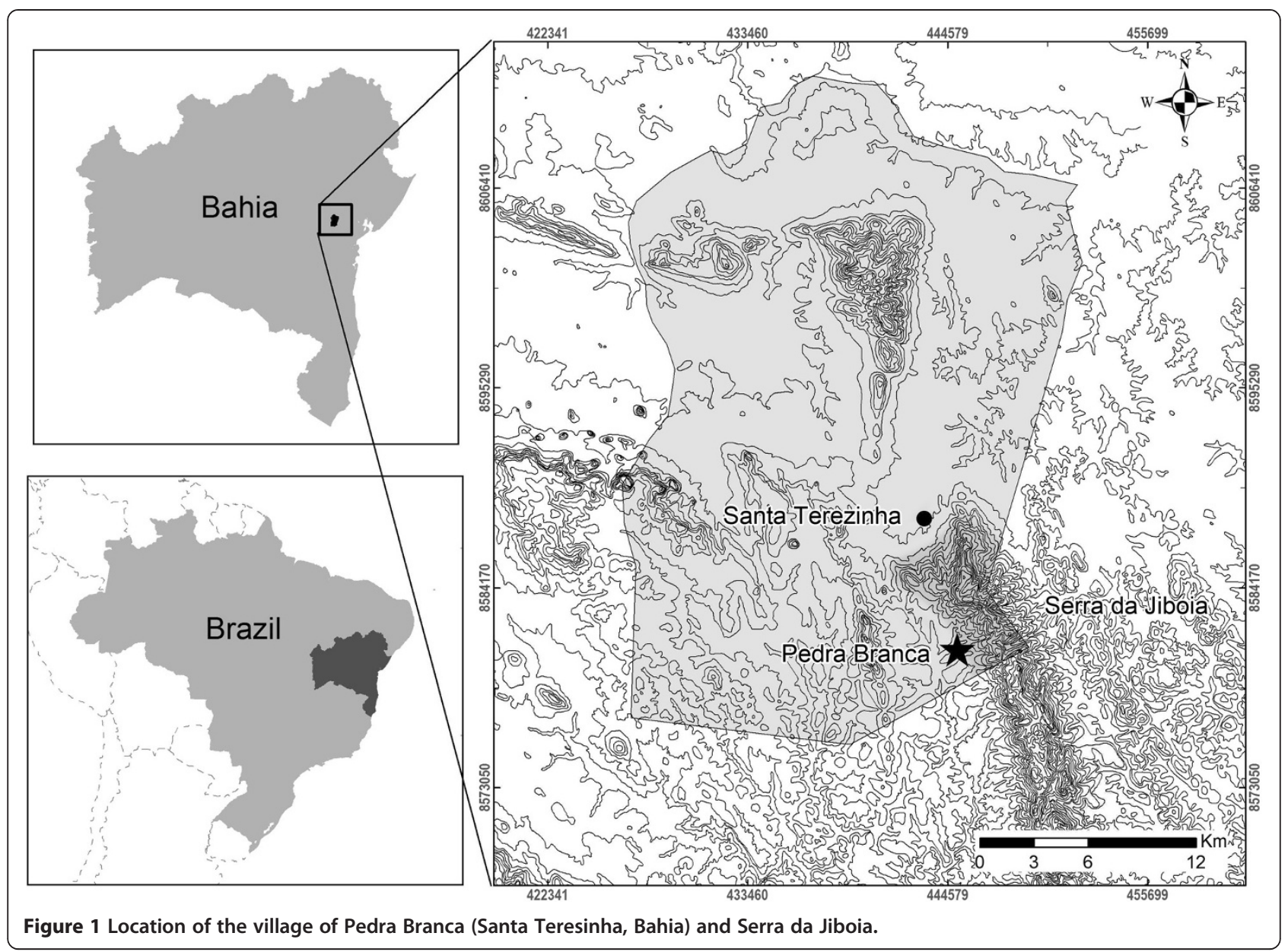

to speak spontaneously about what they saw and heard [34]. These photographs and vocalizations were of species of birds that inhabit both Atlantic Forest and Caatinga environs. The photographs used came from personal files and Wikiaves [35], while the auditory resources came from Wikiaves' data bank. Five male informants (ages 31-63), who showed a more detailed knowledge on the local avifauna during the interviews, were chosen for this data collecting technique. They were approached individually and were very skillful on identifying bird species.

The term ethnospecies is employed in this study when an ethnobiological taxonomic category corresponds to the Linnaean scientific species, independently of how many popular names it receives. The scientific nomenclature followed the Comitê Brasileiro de Registros Ornitológicos [36]. The endangered status followed the Lista Vermelha das Espécies Ameaçadas do Ministério do Meio Ambiente [37], the International Union of Conservation of Nature [38], and the Birdlife International [39].

Data were analyzed using the union model. According to this model, all available information on the surveyed subject is to be considered [40]. For checking their reliability, interviews were conducted both in synchronous situations, with the same question being asked to different individuals in a short interval of time, and diachronic situations, when the same question was asked to the same individual in a long time interval [41].

All ethnographic materials (recordings, transcripts, photographs, and drawings) are kept at the Laboratory of Ethnobiology and Ethnoecology of the Universidade Estadual de Feira de Santana (UEFS), state of Bahia, for evidential purposes.

This study adopted the hierarchy structure proposed by Berlin [4], whose decreasing inclusion taxonomic classes form the following levels: kingdom, life form, intermediate, generic, specific, and variety. We used Venn's diagram $[42,43]$ for relating the ethnobiological taxonomy to the Linnaean taxonomy, where there is indication of Linnaean and ethnobiological taxa by means of circles with different marks, and it also enables showing the closeness between folk members [4]. For comparing the popular taxonomy to the Linnaean taxonomy, we used correspondence categories in evaluations regarding the 
popular generic names and scientific species, such as 1:1 correspondence, where the popular generic name refers to a single scientific species; over-differentiation, when two or more generic taxa refer to a scientific species; and under-differentiation may have two types, type 1 occurs when a single generic name refers to two or more species from the same scientific genus and type 2 occurs when a single generic name refers to two or more species from different scientific genera [44].

\section{Results and discussion}

\section{Name formation and identification of ethnospecies}

The interviewed cited 139 common names of wild birds, which refer to 117 Linnaean species (Table 1). Unlike the Berlinean system [4], when the author says that the name structure of specific taxa in the ethnobiological classification systems is regularly binomial, out of the total number of common names in this study, 63 have binomial names and 77 have monomial names. See Table 2 to check out the glossary of all local bird names.

Most of the bird species were given a single name (even when it is a compound name such as beija-flor = hummingbird), although they represent one or more Linnaean species. In other cases, some species are popularly known with different popular names but they correspond to just one Linnaean species. Considering the relationship between generic taxa and scientific species, we registered the occurrence of three types of correspondence between the biological and popular classification systems proposed by Berlin et al. [5]. For instance, out of the three types of beija-flor, only one receives a specification, i.e. beija-flor-rabo-de-tesoura (Eupetomena macroura), due to the morphology of its tail. This type is named one-to-one correspondence. The other two species (Phaethornis pretrei and Florisuga fusca) are named only beija-flor, with an under-differentiation of type 2 . The same happens with the popular name garça, which represents two scientific species (Ardea alba and Bubulcus ibis). We observed the occurrence of an over-differentiation for the specific names coruja-amanhã-eu-vou and coruja-bacurau, identified by means of vocalization, referring to a single species Hydropsalis albicollis. The first specific refers to a common singing in this species and is possibly related with the reproductive behavior, while the second one, corujabacurau, refers to its calling [14].

Common name formation in this study follows different criteria, such as morphology (coloring pattern, body shape and size), behavior (vocalization, reproduction, and feeding), habitat, and anthropogenic features (Table 3). However, vocalization and coloring pattern in birds were the criteria used more frequently by respondents and, according to Berlin [44], morphology is one of the main criteria used to designate ethnospecies, as well as to differentiate them.
Usually, species with rather peculiar morphological similarities are not differentiated and they are given the same name [45,46], as in the cases of corujão (Pulsatrix koeniswaldiana; Strix virgata), garça (Ardea alba; Bubulcus ibis), and gavião-rapina (Geranoaetus albicaudatus; Buteo brachyurus). Moreover, feather coloring patterns are morphological features also used by residents to differentiate females from males, and the latter, usually, have feathers with brighter colors, due to sexual selection, that is, the female will choose the male because of its physical features [47].

Vocalization is an important aspect for identifying bird species, that is, the sound emitted by them often becomes their popular name on a local basis. Thus, there is an onomatopoeic name formation $[44,4,48,49,16,50,51]$.

Among behavioral criteria, also stands out name formation through trophic ecology, such as in the case of specific names: gavião-carrapateiro (Milvago chimachima), which feeds on ticks at cattle and horses; gavião-pega-pinto (Rupornis magnirostris), which feeds on chicks of other birds, among them the domestic ones, regarded as easy prey; and gavião-peneirador (Elanus leucurus), which has the habit of hovering against the wind to see its prey [14,52]. Other studies also pointed out hawk grouping as related to feeding behavior $[53,45]$. The ethnospecies urubu-rei (Sarcoramphus papa) was the only one related to the generic name urubu (urubu-cabeça-vermelha e o urubu-preto), which was named due to its feeding behavior, as the existence of a hierarchy between these species has been reported, where other species feed only after urubu-rei [14].

Regarding names related to reproductive behavior, 3 ethnospecies were related to nest formation: cava-chão (Nystalus maculatus), which digs holes in slopes; carregamadeira (Phacellodomus rufifrons), which piles wood fragments; and joão-de-barro (Furnarius rufus), which uses mud $[14,54]$.

Fluvicola negenta has its nomenclatural formation based on the anthropogenic aspect. Culturally, this ethnospecies is related to religious belief, something which defines its importance within the community $[16,55]$, as reported in the following excerpt: "People have to say they gave the name 'lavandeira' because it helped Our Lady to wash clothes" (Mrs. M, 63 years).

There are species designated on a local basis, however, they may be given another name, such as in the case of Vanellus chilensis, which in Pedra Branca is named espantaboiada, but it is also known as quero-quero: "Each region gives its name. Here it is named 'espanta-boiada'. It is also observed in the football field. There, it is called 'quero-quero'" (Mr. E, 48 years). Stauber et al. [56] emphasize the importance of studies and records of regional and local variations in the popular nomenclature used, before this is overcome by the academic nomenclature and even by the media. 
Table 1 List of birds' common names and their respective academic correspondences

\begin{tabular}{|c|c|c|c|}
\hline Local common names & Synonymy & Scientific names & Family \\
\hline Acauã & Cauã & Herpetotheres cachinnans (Linnaeus, 1758) & Falconidae Leach, 1820 \\
\hline Alma-de-gato & & Piaya cayana (Linnaeus, 1766) & Cuculidae Swainson, 1837 \\
\hline Andorinha & & Progne tapera (Vieillot, 1817) & Hirundinidae Rafinesque, 1815 \\
\hline Anu-branco & & Guira guira (Gmelin, 1788) & Cuculidae Swainson, 1837 \\
\hline Anu-preto & & Crotophaga ani Linnaeus, 1758 & Cuculidae Swainson, 1837 \\
\hline Aracuã & & Ortalis guttata (Spix, 1825) & Cracidae Rafinesque, 1815 \\
\hline Araponga & & Procnias nudicollis (Vieillot, 1817) & Cotingidae Bonaparte, 1849 \\
\hline Assanhaço-comum & & Tangara sayaca (Linnaeus, 1766) & Thraupidae Cabanis, 1847 \\
\hline Assanhaço-coqueiro & & Tangara palmarum (Wied, 1823) & Thraupidae Cabanis, 1847 \\
\hline Azulão & & Cyanoloxia brissonii (Lichtenstein, 1823) & Cardinalidae Ridgway, 1901 \\
\hline Azuzinho & & Tersina viridis (Illiger, 1811) & Thraupidae Cabanis, 1847 \\
\hline \multirow[t]{2}{*}{ Beija-flor } & & Phaethornis pretrei (Lesson \& Delattre, 1839) & Trochilidae Vigors, 1825 \\
\hline & & Florisuga fusca (Vieillot, 1817) & Trochilidae Vigors, 1825 \\
\hline Beija-flor-rabo-de-tesoura & & Eupetomena macroura (Gmelin, 1788) & Trochilidae Vigors, 1825 \\
\hline Beija-flor-verde & Martim-pescador & Galbula ruficauda Cuvier, 1816 & Galbulidae Vigors, 1825 \\
\hline Bem-te-vi & Bem-te-vi-coroão & Pitangus sulphuratus (Linnaeus, 1766) & Tyrannidae Vigors, 1825 \\
\hline Bem-te-vi-ciseri & & Megarynchus pitanguá (Linnaeus, 1766) & Tyrannidae Vigors, 1825 \\
\hline Bem-te-vi-menor & & Myiozetetes similis (Spix, 1825) & Tyrannidae Vigors, 1825 \\
\hline Bico-de-lacre & & Estrilda astrild (Linnaeus, 1758) & Estrildidae Bonaparte, 1850 \\
\hline Bigode & & Sporophila lineola (Linnaeus, 1758) & Emberezidae Vigors, 1825 \\
\hline Caboculinho & & Sporophila bouvreuil (Statius Muller, 1776) & Emberezidae Vigors, 1825 \\
\hline Caburé & & Glaucidium brasilianu (Gmelin, 1788) & Strigidae Leach, 1820 \\
\hline Caburé-de-estaca & Caburé-de-murundu & Athene cunicularia (Molina, 1782) & Strigidae Leach, 1820 \\
\hline Caga-cebo & & Todirostrum cinereum (Linnaeus, 1766) & Tyrannoidea Vigors, 1825 \\
\hline Canário-belga & Canário-da-Alemanha & Serinus canária (Linnaeus, 1758) & Fringillidae Leach, 1820 \\
\hline Canário-da-capoura & & Sicalis luteola (Sparrman, 1789) & Emberezidae Vigors, 1825 \\
\hline Canário-da-terra & Canário-comum & Sicalis flaveola (Linnaeus, 1766) & Emberezidae Vigors, 1825 \\
\hline Cancan & & Cyanocorax cyanopogon (Wied, 1821) & Corvidae Leach, 1820 \\
\hline Capitão-de-preá & & Laterallus viridis (Statius Muller, 1776) & Rallidae Rafinesque, 1815 \\
\hline Cardeal & & Paroaria dominicana (Linnaeus, 1758) & Thraupidae Cabanis, 1847 \\
\hline Carrega-madeira & Gué-gué & Phacellodomus rufifrons (Wied, 1821) & Furnariidae Gray, 1840 \\
\hline Casaca-de-couro & Pica-pau & Pseudoseisura cristata (Spix, 1824) & Furnariidae Gray, 1840 \\
\hline Cava-chão & & Nystalus maculatus (Gmelin, 1788) & Bucconidae Horsfield, 1821 \\
\hline Chapéu-de-couro & & Chrysomus ruficapillus (Vieillot, 1819) & Icteridae Vigors, 1825 \\
\hline Charuteira & & Gallinago paraguaiae (Vieillot, 1816) & Scolopacidae Rafinesque, 1815 \\
\hline Chorão & & Sporophila leucoptera (Vieillot, 1817) & Emberezidae Vigors, 1825 \\
\hline Chupa-laranja & Papa-laranja & Coereba flaveola (Linnaeus, 1758) & Coerebidae D'orbigny \& Lafresnaye, 1838 \\
\hline Codorna-pimpão & Codorna-maior & Nothura maculosa (Temminck, 1815) & Tinamidae Gray, 1840 \\
\hline Codorna-piriri & & Nothura boraquira (Spix, 1825) & Tinamidae Gray, 1840 \\
\hline Coleiro & & Sporophila albogularis (Spix, 1825) & Emberezidae Vigors, 1825 \\
\hline Corró & & Taraba major (Vieillot, 1816) & Thamnophilidae Swainson, 1824 \\
\hline Corró-pequeno & & Thamnophilus pelzelni Hellmayr, 1924 & Thamnophilidae Swainson, 1824 \\
\hline Corta-colete & & Tangara cayana (Linnaeus, 1766) & Thraupidae Cabanis, 1847 \\
\hline Coruja & & Hydropsalis albicollis (Gmelin, 1789) & Caprimulgidae Vigors, 1825 \\
\hline
\end{tabular}


Table 1 List of birds' common names and their respective academic correspondences (Continued)

\begin{tabular}{|c|c|c|c|}
\hline & $\begin{array}{l}\text { Coruja-amanhã-eu-vou; } \\
\text { Coruja-bacurau; Bacurau }\end{array}$ & & \\
\hline \multirow[t]{3}{*}{ Corujão } & & Tyto alba (Scopoli, 1769) & Tytonidae Mathews, 1912 \\
\hline & & $\begin{array}{l}\text { Pulsatrix koeniswaldiana } \\
\text { (Bertoni \& Bertoni, 1901) }\end{array}$ & Strigidae Leach, 1820 \\
\hline & & Stix virgata (Cassin, 1849) & Strigidae Leach, 1820 \\
\hline \multirow[t]{2}{*}{ Corujão-de-orelha } & Caburé-de-orelha & Megascops choliba & Strigidae \\
\hline & & (Vieillot, 1817) & Leach, 1820 \\
\hline Coruja-rabo-de-tesoura & Coruja-tô-rica & Hydropsalis torquata (Gmelin, 1789) & Caprimulgidae Vigors, 1825 \\
\hline Cuiuba & & Forpus xanthopterygius (Spix, 1824) & Psittacidae Rafinesque, 1815 \\
\hline Curió & & Sporophila angolensis (Linnaeus, 1766) & Emberezidae Vigors, 1825 \\
\hline Espanta-boiada & Quero-quero & Vanellus chilensis (Molina, 1782) & Charadriidae Leach, 1820 \\
\hline Estevo & Trinca-ferro, Pixarro; Vaqueiro & Saltator similis d'Orbigny \& Lafresnaye, 1837 & Thraupidae Cabanis, 1847 \\
\hline Estrelinha & & Lanio pileatus (Wied, 1821) & Thraupidae Cabanis, 1847 \\
\hline \multirow[t]{2}{*}{ Garça } & & Ardea Alba Linnaeus, 1758 & Ardeidae Leach, 1820 \\
\hline & & Bubulcus íbis (Linnaeus, 1758) & Ardeidae Leach, 1820 \\
\hline Garrincha & & Troglodytes musculus Naumann, 1823 & Troglodytidae Swainson, 1831 \\
\hline Gavião-carcará & & Caracara plancus (Miller, 1777) & Falconidae Leach, 1820 \\
\hline Gavião-carrapateiro & Carcará-pequeno & Milvago chimachima (Vieillot, 1816) & Falconidae Leach, 1820 \\
\hline Gavião-pé-de-morro & & Geranospiza caerulescens (Vieillot, 1817) & Accipitridae Vigors, 1824 \\
\hline Gavião-pedrez & Gavião-pega-pinto & Rupornis magnirostris (Gmelin, 1788) & Accipitridae Vigors, 1824 \\
\hline Gavião-peneira & & Elanus leucurus (Vieillot, 1818) & Accipitridae Vigors, 1824 \\
\hline \multirow[t]{2}{*}{ Gavião-rapina } & & Geranoaetus albicaudatus (Vieillot, 1816) & Accipitridae Vigors, 1824 \\
\hline & & Buteo brachyurus Vieillot, 1816 & Accipitridae Vigors, 1824 \\
\hline Guriatá-verdadeira & & Euphonia violácea (Linnaeus, 1758) & Fringillidae Leach, 1820 \\
\hline Guriatá-vivi & & Euphonia clorotica (Linnaeus, 1766) & Fringillidae Leach, 1820 \\
\hline Jacu-verdadeiro & $\begin{array}{l}\text { Jacu-gogó-vermelho; } \\
\text { Jacu-pemba }\end{array}$ & Penelope superciliaris Temminck, 1815 & Cracidae Rafinesque, 1815 \\
\hline Jesus-meu-Deus & & Zonotrichia capensis (Statius Muller, 1776) & Emberezidae Vigors, 1825 \\
\hline João-de-barro & & Furnarius rufus (Gmelin, 1788) & Furnariidae Gray, 1840 \\
\hline Juriti & & Leptotila verreauxi Bonaparte, 1855 & Columbidae Leach, 1820 \\
\hline Lavandeira & & Fluvicola negenta (Linnaeus, 1766) & Tyrannidae Vigors, 1825 \\
\hline Macuca & & Tinamus solitarius (Vieillot, 1819) & Tinamidae Gray, 1840 \\
\hline Mãe-da-lua & Urutau & Nyctibius griséus (Gmelin, 1789) & Nyctibiidae Chenu \& Des Murs, 1851 \\
\hline Maria-do-dia & & Elaenia flavogaster (Thunberg, 1822) & Tyrannidae Vigors, 1825 \\
\hline \multirow[t]{2}{*}{ Marreca } & Pato-verdadeiro; Pato-d'água & Porphyrio Martinica (Linnaeus, 1766) & Rallidae Rafinesque, 1815 \\
\hline & & Dendrocygna viduata (Linnaeus, 1766) & Anatidae Leach, 1820 \\
\hline Martim-pescador & & Megaceryle torquata (Linnaeus, 1766) & Alcedinidae Rafinesque, 1815 \\
\hline Mergulhão & & Tachybaptus dominicus (Linnaeus, 1766) & Podicipedidae Bonaparte, 1831 \\
\hline Nambu-pé-roxo & & Crypturellus tataupa (Temminck, 1815) & Tinamidae Gray, 1840 \\
\hline Nambu-pé-vermelho & & Crypturellus parvirostris (Wagler, 1827) & Tinamidae Gray, 1840 \\
\hline Papa-arroz & Sangue-de-boi & Sturnella superciliaris (Bonaparte, 1850) & Icteridae Vigors, 1825 \\
\hline Papa-café & & Schistochlamys ruficapillus (Vieillot, 1817) & Thraupidae Cabanis, 1847 \\
\hline Papa-capim & & Sporophila nigricollis (Vieillot, 1823) & Emberezidae Vigors, 1825 \\
\hline Pardal & & Passer domesticus (Linnaeus, 1758) & Passeridae Rafinesque, 1815 \\
\hline Pássaro-preto & & Gnorimopsar chopi (Vieillot, 1819) & Icteridae Vigors, 1825 \\
\hline
\end{tabular}


Table 1 List of birds' common names and their respective academic correspondences (Continued)

\begin{tabular}{|c|c|c|c|}
\hline Pêga & & Icteru pyrrhopterus (Vieillot, 1819) & Icteridae Vigors, 1825 \\
\hline Peixe-frito & Sede-sede & Tapera naevia (Linnaeus, 1766) & Taperinae Verheyen, 1956 \\
\hline Perdiz & & Rhynchotus rufescens (Temminck, 1815) & Tinamidae Gray, 1840 \\
\hline Periquito & & Aratinga cactorum (Kuhl, 1820) & Psittacidae Rafinesque, 1815 \\
\hline \multirow[t]{3}{*}{ Pica-pau } & & Veniliornis passerines (Linnaeus, 1766) & Picidae Leach, 1820 \\
\hline & & Drycopus lineatus (Linnaeus, 1766) & Picidae Leach, 1820 \\
\hline & & Colaptes melanochloros (Gmelin, 1788) & Picidae Leach, 1820 \\
\hline Pintassilgo & & Sporagra yarrellii (Audubon, 1839) & Fringillidae Leach, 1820 \\
\hline Pomba-verdadeira & Pomba-do-sertão & Patagioenas picazuro (Temminck, 1813) & Columbidae Leach, 1820 \\
\hline Pomba-do-Pará & & Não identificado & - \\
\hline Rolinha-branca & & Columbina picui (Temminck, 1813) & Columbidae Leach, 1820 \\
\hline Rolinha-caldo-de-feijão & & Columbina talpacoti (Temminck, 1811) & Columbidae Leach, 1820 \\
\hline Rolinha-fogo-pago & & Columbina squamatta (Lesson, 1831) & Columbidae Leach, 1820 \\
\hline Rolinha-Santo-Antônio & & Columbina minuta (Linnaeus, 1766) & Columbidae Leach, 1820 \\
\hline Sabiá-bico-de-osso & & Turdus amaurochalinus Cabanis, 1850 & Turdidae Rafinesque, 1815 \\
\hline Sabiá-branca & & Turdus leucomela Vieillot, 1818 & Turdidae Rafinesque, 1815 \\
\hline Sabiá-coca & & Turdus rufiventris Vieillot, 1818 & Turdidae Rafinesque, 1815 \\
\hline Sabiá-lasca-carne & & Mimus saturninus (Lichtenstein, 1823) & Mimidae Bonaparte, 1853 \\
\hline Saiacaia & Cavala & Gallinago undulata (Boddaert, 1783) & Scolopacidae Rafinesque, 1815 \\
\hline Sangue-de-boi & & Ramphocelus bresilius (Linnaeus, 1766) & Thraupidae Cabanis, 1847 \\
\hline Saracura & Três-potes; Sete-potes & Aramides cajanea (Statius Muller, 1776) & Rallidae Rafinesque, 1815 \\
\hline Siriema & & Cariama cristata (Linnaeus, 1766) & Cariamidae Bonaparte, 1850 \\
\hline Socó-boi & & Butorides striata (Linnaeus, 1758) & Ardeidae Leach, 1820 \\
\hline Sofrê & & Icterus jamacaii (Gmelin, 1788) & Icteridae Vigors, 1825 \\
\hline Tiotoin & & Synallaxis frontalis Pelzeln, 1859 & Furnariidae Gray, 1840 \\
\hline Tiziu & Biziu & Volatinia jacarina (Linnaeus, 1766) & Emberezidae Vigors, 1825 \\
\hline Tororó & & Não identificado & - \\
\hline Tucano & & Ramphastus vitellinus Lichtenstein, 1823 & Ramphastidae Vigors, 1825 \\
\hline Urubu-da-cabeça-vermelha & Bosteiro & Cathartis aurea (Linnaeus, 1758) & Cathartidae Lafresnaye, 1839 \\
\hline Urubu-preto & Urubu-carniceiro & Coragips atratus (Bechstein, 1793) & Cathartidae Lafresnaye, 1839 \\
\hline Urubu-rei & & Sarcoramphus papa (Linnaeus, 1758) & Cathartidae Lafresnaye, 1839 \\
\hline Viuvinha & & Xolmis irupero (Vieillot, 1823) & Tyrannidae Vigors, 1825 \\
\hline Xanana & & Jacana jacana (Linnaeus, 1766) & Jacanidae Chenu \& Des Murs, 1854 \\
\hline Zabelê & & Crypturellus noctivagus (Wied, 1820) & Tinamidae Gray, 1840 \\
\hline
\end{tabular}

Common names follow the local language.

*Endangered species [37].

In what concerns the field of ornithology, this study corroborates the importance of researching on local names [16], as a new distribution in the occurrence of Strix virgata has been recorded for the state of Bahia, namely at the Serra da Jiboia. This species has few records in Bahia and in accordance with literature its distribution was just known to the south of this northeastern state $[57,14,58,59]$. The latest photographic records are documented in the site Wikiaves [60-62]. In the present study this record just happened because this bird gets the popular name corujão, which also refers to two academic species, Pulsatrix koeniswaldiana and Tyto $a l b a$, and taking into account the morphological resemblance to the first one, Strix virgata was only identified through the collection of a specimen by means of some informers' reports about its position inside the woods. Similar situation was experienced by Sick et al. [63] in the Raso da Catarina (Bahia) when they reported that 
Table 2 Correspondence between bird species common names and scientific names

\begin{tabular}{|c|c|c|}
\hline Local common names & English common names & Espécies científicas \\
\hline Acauã & Laughing Falcon* & Herpetotheres cachinnans (Linnaeus, 1758) \\
\hline Alma-de-gato & Squirrel Cuckoo* & Piaya cayana (Linnaeus, 1766) \\
\hline Andorinha & Brown-chested Martin* & Progne tapera (Vieillot, 1817) \\
\hline Anu-branco & White Anu** & Guira guira (Gmelin, 1788) \\
\hline Anu-preto & Black Anu** & Crotophaga ani Linnaeus, 1758 \\
\hline Aracuã & Speckled Chachalaca* & Ortalis guttata (Spix, 1825) \\
\hline Araponga & Bare-throated Bellbird* & Procnias nudicollis (Vieillot, 1817) \\
\hline Assanhaço-comum & Sayaca Tanager* & Tangara sayaca (Linnaeus, 1766) \\
\hline Assanhaço-coqueiro & Coconut Tanager** & Tangara palmarum (Wied, 1823) \\
\hline Azulão & Big Blue** & Cyanoloxia brissonii (Lichtenstein, 1823) \\
\hline Azuzinho & Little Blue** & Tersina viridis (Illiger, 1811) \\
\hline Bacurau & Night Hawk** & Hydropsalis albicollis (Gmelin, 1789) \\
\hline \multirow[t]{2}{*}{ Beija-flor } & Planalto Hermit* & Phaethornis pretrei (Lesson \& Delattre, 1839) \\
\hline & Black Jacobin* & Florisuga fusca (Vieillot, 1817) \\
\hline Beija-flor-rabo-de-tesoura & Scissor-tailed Hummingbird** & Eupetomena macroura (Gmelin, 1788) \\
\hline Beija-flor-verde & Green Hummingbird** & Galbula ruficauda Cuvier, 1816 \\
\hline Bem-te-vi & Great Kiskadee* & Pitangus sulphuratus (Linnaeus, 1766) \\
\hline Bem-te-vi-ciseri & Kiskadee ${ }^{* *}$ & Megarynchus pitanguá (Linnaeus, 1766) \\
\hline Bem-te-vi-coroão & Crowned Kiskadee** & Pitangus sulphuratus (Linnaeus, 1766) \\
\hline Bem-te-vi-menor & Small Kiskadee** & Myiozetetes similis (Spix, 1825) \\
\hline Bico-de-lacre & Common Waxbill* & Estrilda astrild (Linnaeus, 1758) \\
\hline Bigode & Mustache** & Sporophila lineola (Linnaeus, 1758) \\
\hline Biziu & Blue-black Grassquit* & Volatinia jacarina (Linnaeus, 1766) \\
\hline Bosteiro & Shit-eater** & Cathartis aurea (Linnaeus, 1758) \\
\hline Caboculinho & Copper Seedeater* & Sporophila bouvreuil (Statius Muller, 1776) \\
\hline Caburé & Ferruginous Pygmy-Owl* & Glaucidium brasilianu (Gmelin, 1788) \\
\hline Caburé-de-estaca & Pole Pygmy-Owl** & Athene cunicularia (Molina, 1782) \\
\hline Caburé-de-murundu & Hillock Pygmy-Owl** & Athene cunicularia (Molina, 1782) \\
\hline Caburé-de-orelha & Eared Pygmy-Ow|** & Megascops choliba (Vieillot, 1817) \\
\hline Caga-cebo & Common Tody-Flycatcher* & Todirostrum cinereum (Linnaeus, 1766) \\
\hline Canário-belga & Belgian Canary** & Serinus canária (Linnaeus, 1758) \\
\hline Canário-comum & Saffron Finch* & Sicalis flaveola (Linnaeus, 1766) \\
\hline Canário-da-Alemanha & German Canary** & Serinus canária (Linnaeus, 1758) \\
\hline Canário-da-capoura & Barton Canary** & Sicalis luteola (Sparrman, 1789) \\
\hline Canário-da-terra & Earthy Canary** & Sicalis flaveola (Linnaeus, 1766) \\
\hline Cancan & White-naped Jay* & Cyanocorax cyanopogon (Wied, 1821) \\
\hline Capitão-de-preá & Russet-crowned Crake* & Laterallus viridis (Statius Muller, 1776) \\
\hline Cardeal & Red-cowled Cardinal* & Paroaria dominicana (Linnaeus, 1758) \\
\hline Carrega-madeira & Loading timber** & Phacellodomus rufifrons (Wied, 1821) \\
\hline Casaca-de-couro & Leather jacket** & Pseudoseisura cristata (Spix, 1824) \\
\hline Cava-chão & Floor Digger** & Nystalus maculatus (Gmelin, 1788) \\
\hline Cavala & Mackerel** & Gallinago undulata (Boddaert, 1783) \\
\hline Chapéu-de-couro & Chestnut-capped Blackbird* & Chrysomus ruficapillus (Vieillot, 1819) \\
\hline Charuteira & Cigarette Case ${ }^{* *}$ & Gallinago paraguaiae (Vieillot, 1816) \\
\hline
\end{tabular}


Table 2 Correspondence between bird species common names and scientific names (Continued)

\begin{tabular}{|c|c|c|}
\hline Chorão & Crybaby** & Sporophila leucoptera (Vieillot, 1817) \\
\hline Chupa-laranja & Orange Sucker** & Coereba flaveola (Linnaeus, 1758) \\
\hline Codorna-maior & Large Quail** & Nothura maculosa (Temminck, 1815) \\
\hline Codorna-pimpão & Crucian Quail** & Nothura maculosa (Temminck, 1815) \\
\hline Codorna-piriri & Piriri Quail** & Nothura boraquira (Spix, 1825) \\
\hline Coleiro & Collared Seedeater** & Sporophila albogularis (Spix, 1825) \\
\hline Corró & Great Antshrike* & Taraba major (Vieillot, 1816) \\
\hline Corró-pequeno & Little Antshrike** & Thamnophilus pelzelni Hellmayr, 1924 \\
\hline Corta-colete & Burnished-buff Tanager* & Tangara cayana (Linnaeus, 1766) \\
\hline Coruja & Owl** & Hydropsalis albicollis (Gmelin, 1789) \\
\hline Coruja-amanhã-eu-vou & Tomorrow-I-will Owl** & Hydropsalis albicollis (Gmelin, 1789) \\
\hline Coruja-bacurau & Night Hawk Owl** & Hydropsalis albicollis (Gmelin, 1789) \\
\hline \multirow[t]{3}{*}{ Corujão } & Big Owl $\left.\right|^{* *}$ & Tyto alba (Scopoli, 1769) \\
\hline & & Pulsatrix koeniswaldiana (Bertoni \& Bertoni, 1901) \\
\hline & & Stix virgata (Cassin, 1849) \\
\hline Corujão-de-orelha & Eared Owl** & Megascops choliba (Vieillot, 1817) \\
\hline Coruja-rabo-de-tesoura & Scissor-tailed Owl** & Hydropsalis torquata (Gmelin, 1789) \\
\hline Coruja-tô-rica & Wealthy Owl** & Hydropsalis torquata (Gmelin, 1789) \\
\hline Cuiuba & Blue-winged Parrotlet* & Forpus xanthopterygius (Spix, 1824) \\
\hline Curió & Chestnut-bellied Seed-Finch* & Sporophila angolensis (Linnaeus, 1766) \\
\hline Espanta-boiada & Cattle Bugaboo** & Vanellus chilensis (Molina, 1782) \\
\hline Estevo & Green-winged Saltator* & Saltator similis d'Orbigny \& Lafresnaye, 1837 \\
\hline Estrelinha & Pileated Finch* & Lanio pileatus (Wied, 1821) \\
\hline \multirow[t]{2}{*}{ Garça } & Great Egret* & Ardea Alba Linnaeus, 1758 \\
\hline & Cattle Egret* & Bubulcus íbis(Linnaeus, 1758) \\
\hline Garrincha & Southern House Wren* & Troglodytes musculus Naumann, 1823 \\
\hline Gavião-carcará & Caracara Hawk** & Caracara plancus (Miller, 1777) \\
\hline Gavião-carrapateiro & Tick-eater Hawk** & Milvago chimachima (Vieillot, 1816) \\
\hline Gavião-pé-de-morro & Foot-of-the-hill Hawk* & Geranospiza caerulescens (Vieillot, 1817) \\
\hline Gavião-pedrez & Check Hawk** & Rupornis magnirostris (Gmelin, 1788) \\
\hline Gavião-pega-pinto & Chick-Catcher Hawk** & Rupornis magnirostris (Gmelin, 1788) \\
\hline Gavião-peneira & Strainer Hawk** & Elanus leucurus (Vieillot, 1818) \\
\hline Gavião-rapina & Rapine Hawk** & Geranoaetus albicaudatus (Vieillot, 1816) Buteo brachyurus Vieillot, 1816 \\
\hline Gué-gué & Rufous-fronted Thornbird* & Phacellodomus rufifrons (Wied, 1821) \\
\hline Guriatá-verdadeira & True Guriatá** & Euphonia violacea (Linnaeus, 1758) \\
\hline Guriatá-vivi & Guriatá** & Euphonia clorotica (Linnaeus, 1766) \\
\hline Jacu-gogó-vermelho & Red throate Guan** & Penelope superciliaris Temminck, 1815 \\
\hline Jacu-pemba & Pemba Guan** & Penelope superciliaris Temminck, 1815 \\
\hline Jacu-verdadeiro & Rusty-margined Guan* & Penelope superciliaris Temminck, 1815 \\
\hline Jesus-meu-Deus & Rufous-collared Sparrow* & Zonotrichia capensis (Statius Muller, 1776) \\
\hline João-de-barro & Rufous Hornero* & Furnarius rufus (Gmelin, 1788) \\
\hline Juriti & White-tipped Dove* & Leptotila verreauxi Bonaparte, 1855 \\
\hline Lavandeira & Washer** & Fluvicola negenta (Linnaeus, 1766) \\
\hline Macuca & Solitary Tinamou* & Tinamus solitarius (Vieillot, 1819) \\
\hline Mãe-da-lua & Moon Mother** & Nyctibius griséus (Gmelin, 1789) \\
\hline
\end{tabular}


Table 2 Correspondence between bird species common names and scientific names (Continued)

\begin{tabular}{|c|c|c|}
\hline Maria-do-dia & Day Mary** & Elaenia flavogaster (Thunberg, 1822) \\
\hline \multirow[t]{2}{*}{ Marreca } & Purple Gallinule* & Porphyrio Martinica (Linnaeus, 1766) \\
\hline & White-faced Whistling-Duck & Dendrocygna viduata (Linnaeus, 1766) \\
\hline Martim-pescador & Kingfisher** & Megaceryle torquata (Linnaeus, 1766) \\
\hline Martim-pescador & Kingfisher** & Galbula ruficauda Cuvier, 1816 \\
\hline Mergulhão & Diver** & Tachybaptus dominicus (Linnaeus, 1766) \\
\hline Nambu-pé-roxo & Purple-foot Tinamou** & Crypturellus tataupa (Temminck, 1815) \\
\hline Nambu-pé-vermelho & Red-foot Tinamou** & Crypturellus parvirostris (Wagler, 1827) \\
\hline Papa-arroz & Rice-eater** & Sturnella superciliaris (Bonaparte, 1850) \\
\hline Papa-café & Coffee-eater** & Schistochlamys ruficapillus (Vieillot, 1817) \\
\hline Papa-capim & Grass-eater** & Sporophila nigricollis (Vieillot, 1823) \\
\hline Papa-laranja & Orange-eater** & Coereba flaveola (Linnaeus, 1758) \\
\hline Pardal & Sparrow** & Passer domesticus (Linnaeus, 1758) \\
\hline Pássaro-preto & Black Bird** & Gnorimopsar chopi (Vieillot, 1819) \\
\hline \multirow[t]{2}{*}{ Pato-d'água } & Water Duck* & Porphyrio Martinica (Linnaeus, 1766) \\
\hline & & Dendrocygna viduata (Linnaeus, 1766) \\
\hline \multirow[t]{2}{*}{ Pato-verdadeiro } & True Duck* & Porphyrio Martinica (Linnaeus, 1766) \\
\hline & & Dendrocygna viduata (Linnaeus, 1766) \\
\hline Pêga & Magpie ${ }^{* *}$ & Icteru pyrrhopterus (Vieillot, 1819) \\
\hline Peixe-frito & Fried Fish** & Tapera naevia (Linnaeus, 1766) \\
\hline Perdiz & Red-winged Tinamou* & Rhynchotus rufescens (Temminck, 1815) \\
\hline Periquito & Parakeet $^{* *}$ & Aratinga cactorum (Kuhl, 1820) \\
\hline \multirow[t]{4}{*}{ Pica-pau } & Woodpecker** & Veniliornis passerines (Linnaeus, 1766) \\
\hline & & Drycopus lineatus (Linnaeus, 1766) \\
\hline & & Colaptes melanochloros (Gmelin, 1788) \\
\hline & & Pseudoseisura cristata (Spix, 1824) \\
\hline Pintassilgo & Yellow-faced Siskin* & Sporagra yarrellii (Audubon, 1839) \\
\hline Pixarro & Green-winged Saltator* & Saltator similis d'Orbigny \& Lafresnaye, 1837 \\
\hline Pomba-do-Pará & Pará's Dove** & Não identificado \\
\hline Pomba-do-sertão & Sertão Dove** & Patagioenas picazuro (Temminck, 1813) \\
\hline Pomba-verdadeira & True Dove** & Patagioenas picazuro (Temminck, 1813) \\
\hline Quero-quero & Want-want ${ }^{* *}$ & Vanellus chilensis (Molina, 1782) \\
\hline Rolinha-branca & White Turtledove** & Columbina picui (Temminck, 1813) \\
\hline Rolinha-caldo-de-feijão & Bean soup Turtledove** & Columbina talpacoti (Temminck, 1811) \\
\hline Rolinha-fogo-pago & & Columbina squamatta (Lesson, 1831) \\
\hline Rolinha-Santo-Antônio & Saint Antony Turtledove** & Columbina minuta (Linnaeus, 1766) \\
\hline Sabiá-bico-de-osso & Boned-beak Thrush** & Turdus amaurochalinus Cabanis, 1850 \\
\hline Sabiá-branca & White Thrush ** & Turdus leucomela Vieillot, 1818 \\
\hline Sabiá-coca & Coca Thrush** & Turdus rufiventris Vieillot, 1818 \\
\hline Sabiá-lasca-carne & Chipping-meat Thrush** & Mimus saturninus (Lichtenstein, 1823) \\
\hline Saiacaia & Skirtfall** & Gallinago undulata (Boddaert, 1783) \\
\hline \multirow[t]{2}{*}{ Sangue-de-boi } & Cattle Blood** & Ramphocelus bresilius (Linnaeus, 1766) \\
\hline & & Schistochlamys ruficapillus (Vieillot, 1817) \\
\hline Saracura & Gray-necked Wood-Rail* & Aramides cajanea (Statius Muller, 1776) \\
\hline Sede-sede & Thirst-thirst** & Tapera naevia (Linnaeus, 1766) \\
\hline
\end{tabular}


Table 2 Correspondence between bird species common names and scientific names (Continued)

\begin{tabular}{lll}
\hline Sete-potes & Seven Pots** & Aramides cajanea (Statius Muller, 1776) \\
Siriema & Red-legged Seriema* & Cariama cristata (Linnaeus, 1766) \\
Socó-boi & Striated Heron* & Butorides striata (Linnaeus, 1758) \\
Sofrê & Sufferer** & Icterus jamacaii (Gmelin, 1788) \\
Tiotoin & Sooty-fronted Spinetail* & Synallaxis frontalis Pelzeln, 1859 \\
Tiziu & Blue-black Grassquit* & Volatinia jacarina (Linnaeus, 1766) \\
Tororó & \multicolumn{1}{c}{ - - } & Not identified \\
Três-potes & Three Pots** & Aramides cajanea (Statius Muller, 1776) \\
Trinca-ferro & Green-winged Saltator* & Saltator similis d'Orbigny \& Lafresnaye, 1837 \\
Tucano & Toucan** & Ramphastus vitellinus Lichtenstein, 1823 \\
Urubu-carniceiro & Butcher Vulture** & Cathartis áurea (Linnaeus, 1758) \\
Urubu-da-cabeça-vermelha & Red-headed Vulture** & Cathartis áurea (Linnaeus, 1758) \\
Urubu-preto & Black Vulture** & Coragips atratus (Bechstein, 1793) \\
Urubu-rei & King Vulture** & Sarcoramphus papa (Linnaeus, 1758) \\
Urutau & Common Potoo* & Nyctibius griséus (Gmelin, 1789) \\
Vaqueiro & Cowboy** & Saltator similis d'Orbigny \& Lafresnaye, 1837 \\
Viuvinha & Little Widow** & Xolmis irupero (Vieillot, 1823) \\
Xanana & Wattled Jacana* & Jacana jacana (Linnaeus, 1766) \\
Zabelê & Yellow-legged Tinamou* & Crypturellus noctivagus (Wied, 1820) \\
\hline
\end{tabular}

$(*)$ refers to English common names as cited in the scientific literature; $\left(^{* *}\right)$ refers to those English common names freely translated by the authors.

the participation of local informants was decisive for the record Anodhorhyncus leari.

\section{Ethnotaxonomic classification}

For starting the identification of ethnospecies by informants, we defined two lexemes: ave and pássaro (=passarinho). According to local perception, presence of feathers, presence of wings, being able to fly, as well as body size and breeding for slaughter, were relevant features for this differentiation. The term ave mainly refers to species bred at home, such as galinha (Gallus gallus), pato (Cairina moschata), and sacué (Numida meleagris). In turn, pássaro or passarinho are terms used to designate species that are not domesticated, even those bred in captivity. A testimony exemplifies this semantic distinction: "Ave' is bred at home and 'pássaro' always lives in another place.

Table 3 Common name formation and synonyms of bird species recorded in the village of Pedra Branca (Santa Teresinha, Bahia)

\begin{tabular}{|c|c|}
\hline Nomenclatural criteria & Common names/Synonymy \\
\hline \multicolumn{2}{|l|}{ Morphologic } \\
\hline Color & $\begin{array}{l}\text { Anu-branco, anu-preto, azulão, beija-flor-verde, gavião-pedrez, jacu-gogó-vermelho, nambu-pé-roxo, } \\
\text { nambu-pé-vermelho, pássaro-preto, rolinha-branca, rolinha-caldo-de-feijão, sabiá-bico-de-osso, } \\
\text { sabiá-branca, sangue-de-boi, urubu-preto, urubu-cabeça-vermelha. }\end{array}$ \\
\hline Size & Bem-te-vi-menor, codorna-maior, gavião-carcará-menor, corujão. \\
\hline Body shape & $\begin{array}{l}\text { Beija-flor-rabo-de-tesoura, bem-te-vi-coroão, bigode, coleiro, codorna-pimpão, caburé-de-orelha, } \\
\text { corujão-de-orelha, coruja-rabo-de-tesoura, charuteira. }\end{array}$ \\
\hline Habitat & $\begin{array}{l}\text { Assanhaço-coqueiro, caburé-de-estaca, caburé-de-murundu, canário-da-capoura, canário-da-terra, } \\
\text { canário-belga, espanta-boiada, pato-d'água. }\end{array}$ \\
\hline \multicolumn{2}{|l|}{ Behavior } \\
\hline Reproduction & Cava-chão, carrega-madeira, joão-de-barro, viuvinha. \\
\hline Vocalization & $\begin{array}{l}\text { Acauã, bacurau, bem-te-vi, cancan, cavala, coruja-amanhã-eu-vou, coruja-bacurau, gué-gué, chorão, } \\
\text { guriatá-vivi, jesus-meu-deus, maria-do-dia, peixe-frito, sede-sede, rolinha-fogo-apagou, saiacaia, } \\
\text { socó-boi, tiotoin, trinca-ferro, tiziu, três-potes. }\end{array}$ \\
\hline Feed & $\begin{array}{l}\text { Beija-flor, chupa-laranja, papa-laranja, gavião-carrapateiro, gavião-pega-pinto, gavião-peneira, } \\
\text { martim-pescador, mergulhão, papa-capim, pica-pau, sabiá-lasca-carne, sangue-de-boi, urubu-rei.. }\end{array}$ \\
\hline Anthropogenic aspect & Lavandeira. \\
\hline
\end{tabular}


'Galinha,' 'peru' are called 'aves' and the animals we see flying out there are called 'pássaros"' (Mr. R, 69 years).

The presence of feathers leads some informants to include the term pássaro into ave, but it is differentiated due to the species habitat, either on trees or on the ground: "All feathered 'pássaros' are called 'ave,' you know. All of them are this kind of 'ave'. The difference is subtle, because the difference of 'galinha' is that it lives on the ground, and 'passarinho' does not, it lives over there, it flies, it also sits on the floor, but 'galinha' lives on the ground. 'Passarinhos' build their nests on trees and 'galinhas' on the floor" (Mr. J, 48 years).

Other studies have also reported these categories, and the ethnospecies galinha, peru, and periquito are included as "bred at home" or "bird on the ground", as gavião, garça, and pássaro-preto are "wild bird", "selfbred bird", or "flying bird", and these categories include species that are called pássaros or passarinhos, due to captive breeding [64-66].

For some communities, this category of aves covers animals that fly, present a beak, feathers, and lay eggs $[19,16]$. Brown [67] introduces ave as large animals, which have wings, feathers, and a beak (always including pássaros), whereas the bird life form includes flying mammals, such as morcegos, a fact also found out by Jensen [19] and Blumer [68]. This inclusion is registered in Pedra Branca, as observed in the following interview excerpt: "I think 'morcego' is regarded as 'passarinho', it has wings and flies" (Mr. F, 31 years).

Ethnotaxonomic information of respondents seemingly allows us to order them hierarchically, according to the principles of categorization proposed by Berlin [4], where three hierarchical levels were recognized: life form, generic, and specific. In this study, the lexemes pássaro and passarinho match the ethnotaxonomic level "life form". These lexemes include all wild birds cited in the study, but they do not correspond to the Linnaean taxonomy, because pássaros are animals belonging only to the Passeriformes order [69,70].

The recognition and grouping of the generic name sabiá in the region, for instance, mainly relies on morphology, and the vocalization and trophic criteria are responsible for identifying and defining the specific names (Figure 2). Even though sounds are similar, we can identify species in the field [14,53]. Grouping the specific names sabiá-bicode-osso, sabiá-branca, and sabiá-coca corresponds to the Turdidae family in the Linnaean taxonomy, while the specific name sabiá-lasca-carne belongs to the Linnaean Mimidae family. The correspondences found were 1:1 for the 4 specific names cited.

According to respondents' perception, acauã (Herpetotheres cachinnans) is recognized as a kind of gavião, but it is not included in this generic name, constituting a monotypic generic name. The generic name gavião has eight specific

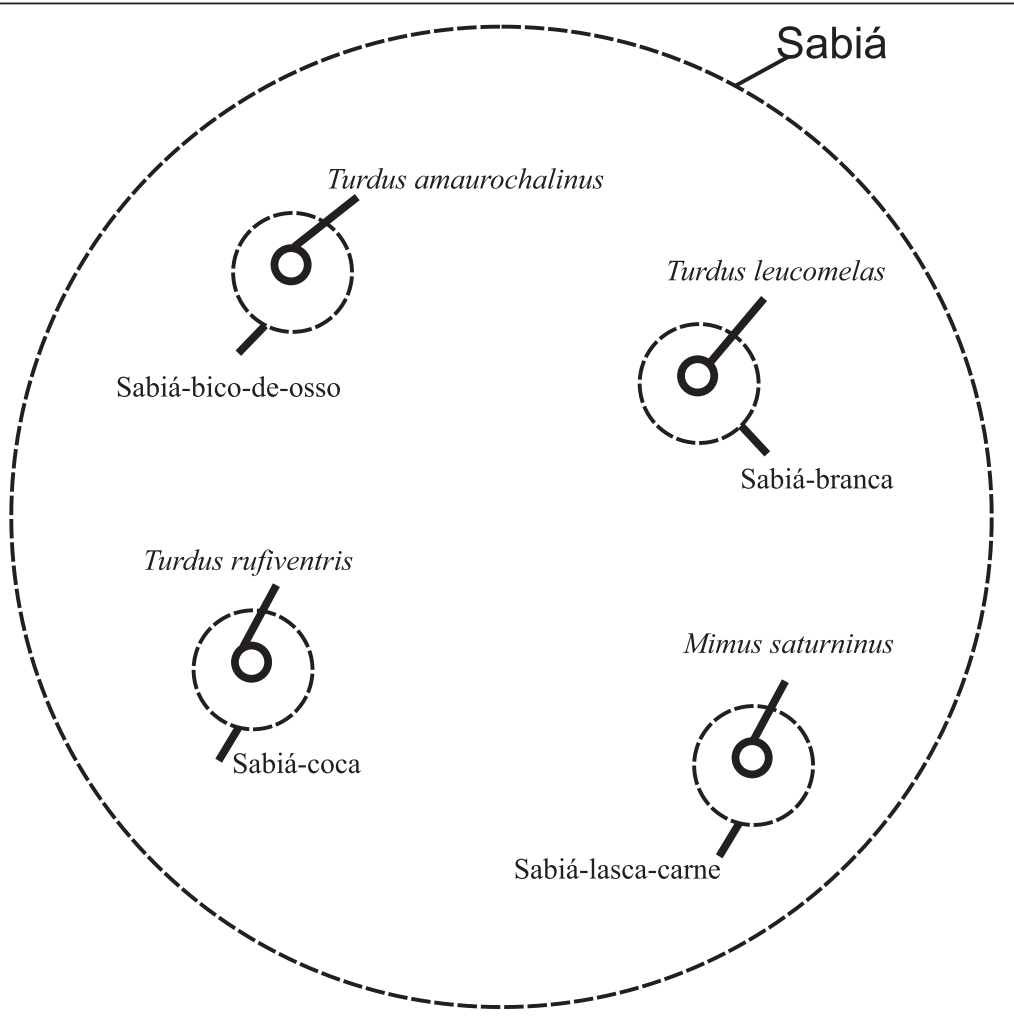

Figure 2 Specific folk generic "sabiá" and its equivalents in academic taxonomy. 


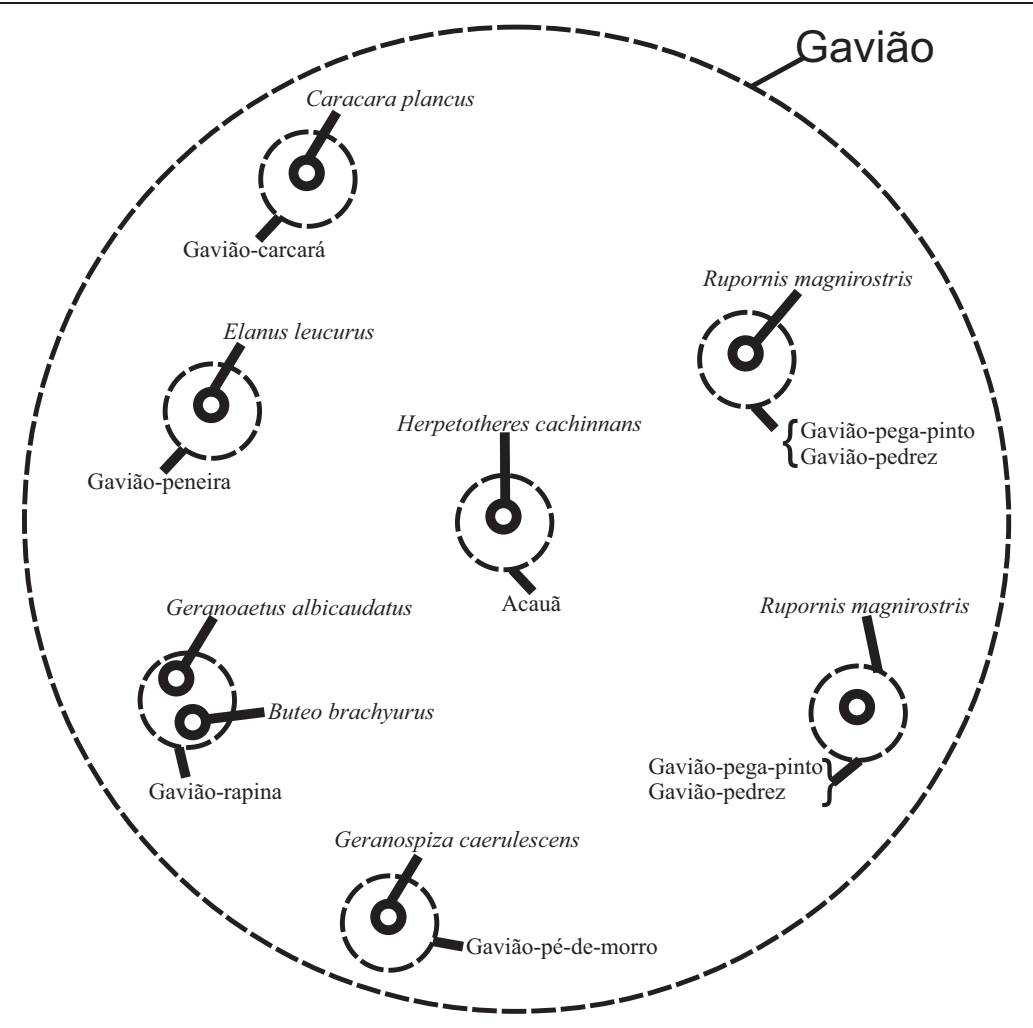

Figure 3 Specific folk generic "gavião" and its equivalents in academic taxonomy.

names and the morphological and behavioral criteria are used to group this set of birds (Figure 3). The specific names gavião-carcará (Caracara plancus), gavião-carrapateiro (synonym gavião-carcará-menor, Milvago chimachima), and acauã (H. cachinnans) correspond to the Falconidae family, and the other ones belong to the Accipitridae family. In this example, 4 1:1 correspondences, 2 under-differentiations, and 1 type $\mathrm{B}$ over-differentiations are found.

\section{Final remarks}

The information registered here shows a broad ethnoornithological knowledge within the community living in the village of Pedra Branca, so that many birds are given names followed by synonyms, while others have only a single name and there is a correspondence between the folk ethnotaxonomy and Linnaean taxonomy.

The results reinforce the need of the participation of local informants in inventories of birds in order to record potential new distributions in the occurrence of species, mainly those nocturnal and even migratory birds.

Through the analysis of popular names, it was possible to identify the nomenclatural criteria used to designate the birds; vocalization and coloring pattern were those more frequently used. Virtually all respondents reported the same etymology for the common names of ethnospecies, something which means that local names are strongly conveyed within the community.

Feeding behavior was a relevant aspect in this research, because besides forming names, it also allowed identifying and hierarchically ordering species. However, the formation of some names did not follow any criteria, as some ethnospecies were identified due to some kind of behavior, vocalization, and habitat.

According to the Berlinean classification model, there was a hierarchy into three levels: life form, generic, and specific. Even meeting two lexemes ave and pássaro (passarinho), we opted to use pássaro at the life form level, listing only the wild birds.

\section{Competing interests}

The authors declare that they have no competing interests.

\section{Authors' contributions}

ATGL carried out the field research and drafted the manuscript. EMCN, CGM, and FMF participated in its design and coordination, and helped to draft the manuscript. All authors read and approved the final manuscript.

\section{Acknowledgments}

The authors thank the community in Pedra Branca, especially the informants, for their information and availability to participate in this study. The biologist Osmar Borges for considerations on the species Strix virgata. The laboratories of Ethnobiology, Ethnoecology, and Ornithology of Feira de Santana State University (UEFS), for their structural and logistical support. The Coordination for the Improvement of Higher Education Personnel (CAPES), for the scholarship granted to the first author. Rafael Almeida for the english review. This article was part of the dissertation entitled "Ethno-ornithology in the 
village of Pedra Branca, Santa Teresinha, Bahia, Brazil", pursued at UEFS' Graduate Program in Zoology.

\section{Author details}

${ }^{1}$ Post-Graduation Program in Zoology, Department of Biological Sciences, Feira de Santana State University, Feira de Santana, Bahia 44036-900, Brazil. ${ }^{2}$ Department of Biological Sciences, Feira de Santana State University, Feira de Santana, Bahia, Brazil.

Received: 13 February 2014 Accepted: 30 June 2014

Published: 10 July 2014

\section{References}

1. Hunn E: The utilitarian factor in folk biological classification. Am J Phys Anthropol 1982, 84:830-847.

2. Hays TE: Ndumba folk biology and general principles of ethnobotanical classifications and nomenclature. Am J Phys Anthropol 1983, 85:592-611.

3. Brown $\mathrm{CH}$ : Mode of subsistence and folk biological taxonomy. Foreign Policy 1985, 26:43-64.

4. Berlin B: Ethnobiological classification: principles of categorization of plants and animals in traditional societies. Princeton: Princeton University Press, Nova Jersey; 1992:1992.

5. Berlin B, Breedlove DE, Raven PH: General principles of classification and nomenclature in folk biology. Am J Anthropol 1973, 75:214-242.

6. Posey DA: Temas e inquirições em etnoentomologia: algumas sugestões quanto à geração de hipóteses. Bol Mus Par Emil Goeld 1987, 3(2):99-134.

7. Farias GB, Alves AGC: Aspectos históricos e conceitos da etnoornitologia. Biotemas 2007, 20(1):91-100.

8. Santos-Fita D, Costa-Neto EM, Costa-Neto EM, Santos-Fita D, Vargas-Clavijo M: Sistemas de classificación etnozoológicos. In Manual de Etnozoología: Uma guia teórico-práctica para investigar La interconexíon del ser humano com los animales. Valencia: Tundra Ediciones; 2009.

9. Couto HH: Ecolingüística: estudo das relações entre língua e meio ambiente. Thesaurus Editora: Brasília; 2007.

10. Brown $\mathrm{CH}$ : Language and living things: uniformities in folk classification and naming. New Brunswick: Rutgers University Press; 1984.

11. Atran S: Cognitive foundations of natural history. Londres: Cambridge University Press. Behav Brain Sci 1990, 21(4):547-569.

12. Atran S: Folk biology and the anthropology of science: cognitive universals and cultural particulars. Printed in the United States of America. Behav Brain Sci 1998, 21:547-609.

13. Ellen RF: The cultural relations of classification: an analysis of Nuaulu animal categories from central Seram. Cambridge: Cambridge University Press; 1993.

14. Sick H: Ornitologia Brasileira. Edição Revista e Ampliada por José Fernando Pacheco (coord). Rio de Janeiro: Nova Fronteira; 1997.

15. Cooke WW: Bird nomenclature of the Chippewa Indians. Auk 1884 1(3):242-250.

16. Farias GB, Alves AGC: É importante pesquisar o nome local das aves? Rev Bras Ornitol 2007, 15(3):403-408.

17. Goeldi E: As aves do Brasil. Rio de Janeiro: Livraria Clássica de Alves e Cia; 1894.

18. Teschauer C: Avifauna e flora nos costumes, superstições e lendas brasileiras e americanas. Edição da Livraria do Globo: Porto Alegre; 1925.

19. Jensen AA: Sistema indígena de classificação de aves: aspectos comparativos, ecológicos e evolutivos. Belem: Bol Mus Par Emil Goeld; 1988.

20. Giannini IV: A ave resgatada: a impossibilidade de leveza do ser. Dissertação de mestrado. Universidade de São Paulo: Departamento de Antropologia; 1991.

21. Carrara E: TSI TEWARA: um vôo sobre o Cerrado Xavante. Dissertação de mestrado. Departamento de Antropologia: Universidade de São Paulo; 1997.

22. Instituto Brasileiro de Geografia e Estatística IBGE. [http://www.ibge.gov. br/cidadesat]

23. Centro de Estatística e Informação SEl. [www.sei.ba.gov.br]

24. Costa-Neto EM, Pacheco JM: A construção do domínio etnozoológico "inseto" pelos moradores do povoado de Pedra Branca, Santa Terezinha, Estado da Bahia. Acta Sci Biol Sci 2004, 26(1):81-90.

25. Secretaria de Assistência a Saúde: Sistema de informação de atenção básica. Santa Teresinha, Bahia: Secretaria Municipal de Saúde; 2011.

26. Tomasoni MA: Recôncavo Sul Pede Socorro. Universidade do Estado da Bahia. Jornal Intercampos 2000.
27. Centro de Estatística e Informação SEl: Informações básicas dos municípios baianos: recôncavo sul. Salvador: Governo do Estado da Bahia; 1994:543-563.

28. Tomasoni MA, Dias S: Lágrimas da Serra: os impactos das atividades agropecuárias sobre o geossistema da APA Municipal da Serra da Jiboia, município de Elisio Medrado, Bahia. [resumo] X Simpósio Brasileiro e Geografia Física Aplicada; 2003 [http://www.cibergeo.org/XSBGFA/eixo3/3.3/ 336/336.htm]

29. Queiroz LP, Sena TSN, Costa MJSL: Flora vascular da Serra da Jiboia, Santa Terezinha, Bahia: o campo rupestre. Feira de Santana. Sitient 1996, 15:27-40.

30. Sobrinho JGC, Queiroz LP: Composição florística de um fragmento de Mata Atlântica na Serra da Jiboia, Santa Terezinha, Bahia, Brasil. Sitient: Série Ciências Biológicas 2005, 5(1):20-28.

31. Valente EB, Porto KS: Hepáticas (Marchantiophyta) de um fragmento de Mata Atlântica na Serra da Jibóia, Município de Santa Teresinha, Bahia, Brasil. Acta Bot Bras 2006, 20(2):433-441.

32. Valente EB, Porto KS, Bôas-Bastos SB, Bastos CJP: Musgos (Bryophyta) de um fragmento de Mata Atlântica na Serra da Jiboia, município de Santa Terezinha, BA, Brasil. Acta Bot Bras 2009, 23(2):369-375.

33. Freitas MA, Moraes EPF: Levantamento da avifauna da Fazendo Jequitibá (Serrada Jiboia), município de Elisio Medrado, Bahia. Atual Ornitol 2009, 147:73-76. Online.

34. Rodrigues AS: Metodología de la investigación etnozoológica. In Manual de Etnozoología: Uma guia teórico-práctica para investigar La interconexíon del ser humano com los animales. Edited by Costa-Neto EM, Santos-Fita D, Vargas-Clavijo M. Valencia: Tundra Ediciones; 2009.

35. Wiki Aves - A Enciclopédia das Aves do Brasi. [http://www.wikiaves.com.br]

36. Comitê Brasileiro de Registros Ornitológicos CBRO. [http://www.cbro.org $\mathrm{br} / \mathrm{CBRO} / \mathrm{pdf} /$ avesbrasil2011.pdf]

37. Ministério do Meio Ambiente MMA: Livro vermelho da fauna vermelha ameaçada de extinção. Brasília: Ministério do Meio Ambiente; 2008.

38. International Union for Conservation of Nature IUCN [http://www.iucnredlist.org.]

39. Bird Life Internetional. [http://www.birdlife.org/datazone/species]

40. Hays TE: An empirical method for the identification of covert categories in ethnobiology. Am Ethnol 1976, 3(3):489-507.

41. Werner O: The basic assumptions of ethnoscience. Semiotica 1969, 01:329-338.

42. Gardner PM: Birds, Words, and a Requiem for the Omniscient Informant. Am Ethnol 1976, 3:446-468.

43. Hunn E: Toward a perceptual model of folk biological classification. Am Ethnol 1976, 3:508-524.

44. Berlin B: Folk Systematics in Relation to Biological Classification and Nomenclature. Annu Rev Ecol Syst 1973, 4:259-271.

45. Gomes CRG, Epifânio AD, Vasconcelos MF: Estudo etnoornitológico no município de Curumbá, Mato Grosso do Sul, Brasil. Atual Ornitol 2010, 158:49-54. on-line.

46. Berlin B, Boster J, O'Neill J: "The perceptual bases of ethnobiological classification: evidence from Aguaruna folk ornithology". J Ethnobiol 1981, 1:95-108.

47. Pough FH, Janis CM, Heiser JB: A Vida dos Vertebrados. 4th edition. Atheneu: São Paulo; 2006.

48. Hunn E: The use of sound recordings as voucher specimens and stimulus materials in ethnozoological research. J Ethnobiol 1992, 12(2):187-198.

49. Ichikawa M: The birds as indicators of the invisible world: Ethno-ornithology of the Mbuti hunter-gatherers. Afr Stud, Supp/ 1998, 25:105-121.

50. Forth G: What's in a Bird's Name: Relationships among Ethno-ornithological Terms in Nage and other Malayo-Polynesian Languages. In Ethno-ornithology: birds, indigenous peoples, culture and society. Edited by Tidermann S, Gosler A. London: Earthscan; 2010:223-237.

51. Berlin B, O'Nell JP: The pervasiveness of onomatopoeia in Aguaruna and Huambisa bird names. J Ethnol 1981, 1(2):238-261.

52. Santos WM, Rosado FR: Dados preliminares da biologia do gavião-carijó (Rupornis magnirostris, Gmelin, 1788) na região Noroeste do Paraná. Revista de Agronegócio e Meio Aambiente 2009, 2(3):421-430.

53. Araujo HFP: Composição da avifauna e etnoornitologia em complexos estuários-manguezais na estado da Paraiba, Brasil. Dissertação de Mestrado. João Pessoa, Paraíba: Universidade Federal da Paraíba, Departamento de Ciências Biológicas; 2005.

54. Narosky T, Carman RL: El honero - ave nacional. Albat: Buenos Aires; 2009. 
55. Farias GB, Alves AGC, Marques JGW: Mythological Relations Between the "Lavandeira" Birds Fluvicola nengeta and Motacilla alba in Northeast Brazil and Northwest Spain: Possible Cultural Implications for Conservation. J Ethnol 2010, 30(2):240-251.

56. Strauber FC, Accordi YA, Argel M: Nomes populares de aves brasileiras coletados por Johann Natterer (1817-1835). Atual Ornitol 2007, 136:1-6.

57. Pinto OMO: Novo catálogo das aves do Brasil. Empresa Gráfica Comércio, Depto. Da Revista dos Tribunais: São Paulo; 1978.

58. Parrini R, Raposo MA, Pacheco JF, Carvalhães AMP, Junior TAM, Fonseca PSM, Minns J: Birds of the Chapada Diamantina. Cotinga: 1999

59. Borges OB: Biogeografia das comunidades de aves das dunas do rio São Francisco, município de Barra/Bahia. Universidade Federal da Bahia, Instituto de Geociências: Monografia de Bacharelado; 2003.

60. Flores FM: Strix virgata (Cassin 1849). [http://www.wikiaves.com/82785]

61. Lemos MS: Strix virgata (Cassin 1849). [http://www.wikiaves.com/828341]

62. Jacomelli: Strix virgata (Cassin 1849). [http://www.wikiaves.com/1009238]

63. Sick H, Gonzaga LP, Teixeira DM: A arara-azul-de-lear, Anodorhynchus Leari Bonaparte, 1856. Rer Bras Zool 1987, 3(7):441-463.

64. Cadima Cl, Marçal-Junior O: Notas sobre etnoornitologia na comunidade do distrito rural de Miraporanga, Uberlandia. Minas Gerais. Bioscience 2004, 20(1):83-94.

65. Santos IB, Costa-Neto EM: Estudo etnoornitológico em uma região do Semi-Árido do estado da Bahia, Brasil. Sitient: Série Ciências Biológicas 2007, 7(3):273-288.

66. Marques JGW: "Do canto bonito ao berro do bode": percepção do comportamento de vocalização em aves entre os camponeses alagoanos. Rev Etol 1998, Número especial:71-85.

67. Brown $\mathrm{CH}$ : Folk zoological life-forms and linguistic marking. J Ethnobiol 1982, 2(1):95-112.

68. Bluner H: Why is the Cassowary Not a Bird? A Problem of Zoological Taxonomy Among the Karamofthe New Guinea Highlands. Man: New Series 1967, 1(2):5-25.

69. Salles O: Nem todas as aves são pássaros. 1986, [http://www.ao.com.br/ aves\&pas.htm]

70. Strauber FC: Todas as aves são pássaros. Atualidades Ornitológicas 2009, $148: 4-6$.

doi:10.1186/1746-4269-10-55

Cite this article as: Galvagne Loss et al:: Ethnotaxonomy of birds by the inhabitants of Pedra Branca Village, Santa Teresinha municipality, Bahia state, Brazil. Journal of Ethnobiology and Ethnomedicine 2014 10:55.

\section{Submit your next manuscript to BioMed Central and take full advantage of:}

- Convenient online submission

- Thorough peer review

- No space constraints or color figure charges

- Immediate publication on acceptance

- Inclusion in PubMed, CAS, Scopus and Google Scholar

- Research which is freely available for redistribution 